\title{
Studies of network organization and dynamics of e-beam crosslinked PVPs: From macro to nano
}

\author{
C. Dispenza ${ }^{\mathrm{a}, \mathrm{b}}$, N. Grimaldi ${ }^{\mathrm{a}, *}$, M.-A. Sabatino $^{\mathrm{a}}$, S. Todaro ${ }^{\mathrm{a}}$, D. Bulone $^{\mathrm{b}}$, D. Giacomazza $^{\mathrm{b}}$, \\ G. Przybytniak ${ }^{\mathrm{c}}$, S. Alessi ${ }^{\mathrm{a}}, \mathrm{G}^{\mathrm{a}}$. Spadaro ${ }^{\mathrm{a}}$ \\ a Dipartimento di Ingegneria Chimica, Gestionale, Informatica, Meccanica, Università degli Studi di Palermo, Viale delle Scienze Ed. 6, 90128 Palermo, Italy \\ ${ }^{\mathrm{b}}$ CNR - Istituto di Biofisica (Palermo unit), Via U. La Malfa 153, 90146 Palermo, Italy \\ ${ }^{\mathrm{c}}$ Centre for Radiation Research and Technology, Institute of Nuclear Chemistry and Technology, Dorodna 16, 03-195 Warsaw, Poland
}

\section{A R T I C L E I N F O}

Article history:

Received 21 September 2011

Accepted 24 November 2011

Available online 3 December 2011

Keywords:

e-Beam irradiation

PVP aqueous solutions

Nanogels

Dynamic mechanical spectroscopy

NMR spin-lattice relaxation

\begin{abstract}
A B S T R A C T
In this work the influence of poly(N-vinyl pyrrolidone) (PVP) concentration in water on the organization and dynamics of the corresponding macro-/nanogel networks has been systematically investigated. Irradiation has been performed at the same irradiation dose (within the sterilization dose range) and dose rate. In the selected irradiation conditions, the transition between macroscopic gelation and micro-/nanogels formation is observed just below the critical overlap concentration ( $\sim 1 \mathrm{wt} \%$ ), whereas the net prevalence of intra-molecular over inter-molecular crosslinking occurs at a lower polymer concentration (below $0.25 \mathrm{wt} \%$ ). Dynamic-mechanical spectroscopy has been applied as a classical methodology to estimate the network mesh size for macrogels in their swollen state, while ${ }^{13} \mathrm{C}$ NMR spin-lattice relaxation spectroscopy has been applied on both the macrogel and nanogel freeze dried residues to withdraw interesting information of the network spatial organization in the passage of scale from macro to nano.
\end{abstract}

(c) 2011 Elsevier Ltd. All rights reserved.

\section{Introduction}

Among the different synthetic methodologies for the production of hydrogels much interest has been paid to the use of e-beam radiation processing, as it generally yields highly pure materials through environmentally friendly and economically viable processes (Rosiak and Olejniczak, 1993; Rosiak et al., 1995; Rosiak and Yoshii, 1999; Lugao and Malmonge, 2001; Chmielewski et al., 2005; Peppas et al., 2006). It is also well known that one of the most appealing features of radiation processing is the possibility of tuning structure and properties of networks obtained from the same feedstock materials through an apt choice of processing parameters (Ulanski and Rosiak, 1999; Henke et al., 2005; An, 2009). As a consequence, convincing structure-process-properties relationships have to be set in place. When gelation is macroscopic, thereby occurring mainly in virtue of inter-molecular crosslinking, the crosslink density is often estimated by means of macroscopic swelling, mechanical or dynamic-mechanical data (Anseth et al., 1996; Kofonas and Cohen, 1997). These methodologies lead to average values of network mesh size that are a convincing description of the network organization only for homogeneous

\footnotetext{
* Corresponding author. Tel.: + 399123863738 ; fax: + 399123860841.

E-mail address: natascia.grimaldi@unipa.it (N. Grimaldi).
}

network structures. This is not often the case of radiation crosslinked networks (D’Errico et al., 2008; Ricca et al., 2010). Furthermore, these conventional characterization methodologies are not suitable for nanogels.

In this work, information on the network organization and dynamics at different length-scales for hydrogels produced by e-beam irradiation from aqueous solutions of a commercial grade poly(N-vinyl-pyrrolidone) (PVP) has been sought. In particular, an irradiation dose of $40 \mathrm{kGy}$, therefore within the sterilization dose range, at controlled low temperature and average dose rate of $30 \mathrm{~Gy} / \mathrm{s}$ has been applied. Different polymer concentrations, from above to below the critical chain overlap concentration for the chosen polymer $(\sim 1 \mathrm{wt} \%)$, have been investigated yielding macrogels and micro-/nanogels. The yield of crosslinking reactions has been estimated gravimetrically and the morphology of the macro/nanogel dry residues has been characterized through scanning electron microscopy (SEM). Conventional swelling and rheological characterizations have been applied to the macrogels, while nanogels' particle size distribution has been determined by dynamic light scattering measurements. A unifying description in terms of network crosslinking density has been attempted through evaluation of spin-lattice relaxation times in the rotating frame $\left(\mathrm{T}_{1} \mathrm{\rho} \mathrm{H}\right)$ from ${ }^{13} \mathrm{C}$ Cross Polarization-Magic Angle Spinning Nuclear Magnetic Resonance $\left({ }^{13} \mathrm{C}\left\{{ }^{1} \mathrm{H}\right\}\right.$ CP-MAS NMR) spectroscopy. 


\section{Experimental}

PVP k60 (Aldrich, $M_{\mathrm{n}}=1.60 \times 10^{5} \mathrm{~g} / \mathrm{mol}, M_{\mathrm{w}}=4.1 \times 10^{5} \mathrm{~g} / \mathrm{mol}$ and radius of gyration, $R_{\mathrm{g}}=27 \mathrm{~nm}$ the last two from static light scattering measurements), was supplied as $45 \mathrm{wt} \%$ aqueous solution. PVP critical chain overlapping concentration in water, $\sim 1 \mathrm{wt} \%$, was determined by intrinsic viscosity measurements and confirmed by laser light scattering measurements (Dispenza et al., 2011).

All solutions were carefully deoxygenated by gaseous nitrogen and bottled in hermetically closed $15 \mathrm{ml}$ glass vials. Solutions at $0.5,0.25,0.1$ and $0.05 \mathrm{wt} \%$ were also saturated by $\mathrm{N}_{2} \mathrm{O}$ prior to irradiation in order to increase the concentration of hydroxyl radicals formed from water radiolysis during irradiation. Electron beam irradiation was performed using LAE 13/9, a $10 \mathrm{MeV}$ linear accelerator of the ICHTJ of Warsaw (Poland) at $0-4{ }^{\circ} \mathrm{C}$. Irradiation conditions were set to have an average current of $0.08 \mu \mathrm{A}$, pulse duration and frequency of $10-12 \mu \mathrm{s}$ and $37.5 \mathrm{~Hz}$, respectively. Total absorbed dose and homogeneous dose distribution along the samples were measured by film polyvinyl chloride dosimeters on a basis of UV-vis absorbance at $396 \mathrm{~nm}$. Macroscopically gelled systems were separated from their soluble portions by immersion for $72 \mathrm{~h}$ in excess double-distilled water at $40{ }^{\circ} \mathrm{C}$ and successively freeze-dried.

After irradiation, samples at $0.5,0.25,0.1$ and $0.05 \mathrm{wt} \%$ were dialyzed against distilled water for $48 \mathrm{~h}$ using dialysis tubes of $100 \mathrm{kDa}$ cut-off (Aldrich). For these systems the yield of the process was determined gravimetrically by comparing the dry weight of the polymer in the sample before and after irradiation followed by dialysis. For PVP solutions at higher concentrations, $10-2 \mathrm{wt} \%$, the gel fractions were determined by Soxhlet extraction for $24 \mathrm{~h}$, using water as solvent. The gel fraction was determined as ratio of the water-insoluble part with respect to the total amount of the dry sample before extraction. The reported results are the average of minimum three independent measurements and the experimental error was $\pm 2 \%$.

The morphology of both macro and nanogels' dried residues has been investigated by a field emission scanning electron microscopy (FESEM) system (JEOL) at an accelerating voltage of $10 \mathrm{kV}$. Swelling measurements were carried out according to an established methodology (Dispenza et al., 2011). Rehydration ratio is defined as $\mathrm{RR}=w_{\mathrm{s}} / w_{\mathrm{d}}$, where $w_{\mathrm{s}}$ and $w_{\mathrm{d}}$ are the measured weights of the hydrogel in the swollen and dry states, respectively. Dynamic-mechanical properties of the hydrogels were assessed by small-amplitude shear experiments. Tests were performed using a Stress-controlled Rheometer Ar 1000TA Instruments with an aluminum plate geometry (diam. $20 \mathrm{~mm}$ ), gap of $1000 \mu \mathrm{m}$ and temperature of $25 \pm 1{ }^{\circ} \mathrm{C}$. The mechanical response of the material is expressed in terms of shear storage or elastic modulus, $G^{\prime}$, and shear loss modulus, $G^{\prime \prime}$, as a function of the frequency. Frequency sweep tests were performed in the LVR at frequency values ranging from 0.01 to $200 \mathrm{rad} / \mathrm{s}$ at the controlled stress of $10 \mathrm{~Pa}$, as determined by preliminary strain sweep tests over the range $1-1000 \mathrm{~Pa}$.

The hydrodynamic radius, $R_{\mathrm{h}}$, of the irradiated PVP samples were measured by dynamic light scattering (DLS) techniques, using a Brookhaven Instruments BI200-SM goniometer. Samples were kept at constant temperature of $20 \pm 0.1{ }^{\circ} \mathrm{C}$. The light scattered intensity and time autocorrelation function were measured using a Brookhaven BI-9000 correlator and a $100 \mathrm{~mW} \mathrm{Ar}$ laser (Melles Griot) tuned at $\lambda=632.8 \mathrm{~nm}$. Measurements were taken at different scattering vectors $q=4 \pi n \lambda / \sin (\theta / 2)$, where $n$ is the refraction index of the solution, $\lambda$ is the wavelength of the incident light and $\theta$ is the scattering angle. Samples were placed in the quartz cell used for the measurement, as produced, after dilution with bidistilled water and/or syringe filtration $(0.8,0.45$ and $0.22 \mu \mathrm{m}$ pore size-Millipore). Because samples showed low polydispersity, dynamic light scattering data were analyzed by the method of cumulants. According to this method the logarithm of the field-correlation function can be written in terms of a polynomial of the delay time $\tau$. This function can be then easily fitted by application of linear least-squares technique. Mean hydrodynamic radius of nanoparticles and standard deviation (the amplitude of size distribution curve) can be calculated accordingly (Stepanek, 1993).

${ }^{13} \mathrm{C}$ Cross Polarization-Magic Angle Spinning Nuclear Magnetic Resonance $\left({ }^{13} \mathrm{C}\left\{{ }^{1} \mathrm{H}\right\} \mathrm{CP}-\mathrm{MAS}\right.$ NMR $)$ spectra were obtained at room temperature through a Bruker Advance II $400 \mathrm{MHz}$ (9.4T) spectrometer operating at $100.63 \mathrm{MHz}$ for the ${ }^{13} \mathrm{C}$ nucleus with a MAS rate of $13 \mathrm{kHz}, 1024$ scans, a contact time of $1.5 \mathrm{~ms}$ and a repetition delay of $4 \mathrm{~s}$. All samples were placed in $4 \mathrm{~mm}$ zirconia rotors with KEL-F caps. The proton spin-lattice relaxation time in the rotating frame $T_{1 \rho} \mathrm{H}$ was determined with the variable spin lock (VSL) pulse sequence using delay times ranging from 0.1 to $7.5 \mathrm{~ms}$ and a contact time of $1.5 \mathrm{~ms}$.

\section{Results and discussion}

At a preliminary visual inspection, the irradiated samples from solutions at 10,8 and $6 \mathrm{wt} \%$ appear completely macroscopically gelled, while at 4,2 and 0.5 wt\% present both macrogel islands and a separated aqueous phase. At lower concentrations, all irradiated systems appear optically transparent liquids and would be "traditionally" classified as "sols".

"Gel fraction" values for macroscopic gels are reported in Table 1 . Systems from 10 to $2 \mathrm{wt} \%$ have similar high gel fraction values (above $85 \%$ ), while the " $0.5 \mathrm{wt} \%$ " system has a lower but still significant gel fraction (about $55 \mathrm{wt} \%$ ) and the sol fraction shows the presence of nanoparticles, as it will be discussed in the following. Mass recovery from dialysis and freeze drying of systems with PVP concentration below $0.5 \mathrm{wt} \%$ was always higher than $90 \%$. SEM morphology of PVP macro and nanogels can be observed in Fig. 1. The dry nanogel particles of the " $0.25 \mathrm{wt} \%$ " system form a thin layer of sub-micron globular aggregates (Fig. 1a). Freeze-dried residues of macrogels obtained at 2, 4 and 8 wt\% are shown in Fig. 1b-d. While at $2 \mathrm{wt} \%$ the freeze dried gels does not present porosity, increasing polymer concentration, up to $6 \mathrm{wt} \%$, a fairly regular porous structure is evidenced. Above this value, porosity is reduced in size with evident heterogeneity in size distribution.

The dynamic storage, $G^{\prime}$, and loss moduli, $G^{\prime \prime}$, as function of frequency are shown in Fig. 2. $G^{\prime}$ and $G^{\prime \prime}$ values at $1 \mathrm{~Hz}$ are also reported in Table 1 . It can be observed that, in the investigated angular frequency range, $G^{\prime}$ values are almost constant and higher than the corresponding $G^{\prime \prime}$ values. This indicates that gels formed by irradiation in these conditions are typical "strong gel", i.e. with prevalent elastic behavior (Anseth et al., 1996). Looking closer at the influence of the polymer concentration, from 2 to $6 \mathrm{wt} \%, G^{\prime}$ increases, while $G^{\prime \prime}$ is almost constant. At higher concentration

\section{Table 1}

Gel fraction, shear storage modulus $\left(G^{\prime}\right)$, shear loss modulus $\left(G^{\prime \prime}\right)$ at $1 \mathrm{rad} / \mathrm{s}$ molecular weight of the polymer chain between two neighboring crosslinking points $\left(M_{\mathrm{c}}\right)$, and network mesh size $(\xi)$, and rehydration ratio at plateau $\left(\mathrm{RR}_{\text {plateau }}\right)$

\begin{tabular}{lllllll}
\hline Pol. conc. (wt\%) & GF (\%) & $\boldsymbol{G}^{\prime}(\mathbf{P a})$ & $\boldsymbol{G}^{\prime \prime}(\mathbf{P a})$ & $\boldsymbol{M}_{\mathbf{c}}(\mathbf{D a})$ & $\boldsymbol{\xi}(\mathbf{n m})$ & $\mathbf{R R}_{\text {plateau }}$ \\
\hline $\mathbf{1 0}$ & 91.2 & 1974 & 21 & 48848 & 11.5 & 25.3 \\
$\mathbf{8}$ & 92.8 & 2811 & 33.5 & 37468 & 11.4 & 27.5 \\
$\mathbf{6}$ & 94 & 3385 & 70 & 28343 & 11.4 & 21.3 \\
$\mathbf{4}$ & 93.5 & 2260 & 76. & 33007 & 12.6 & 20.4 \\
$\mathbf{2}$ & 86 & 1740 & 75 & 32900 & 14.0 & 16.5 \\
\hline
\end{tabular}


a



C

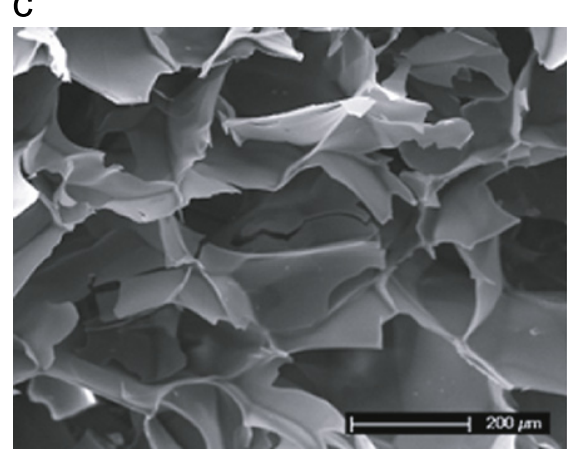

b

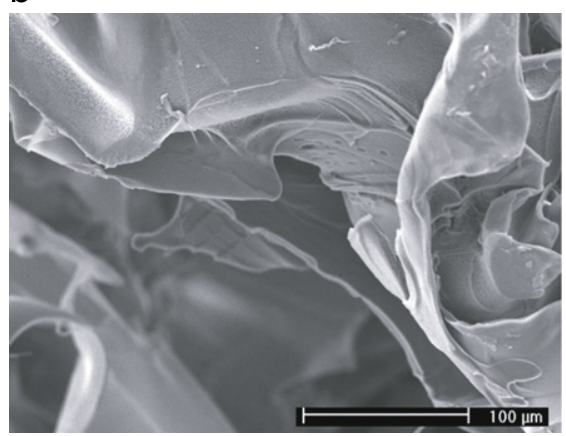

d



Fig. 1. SEM images of dried residues of hydrogels obtained at the variance of polymer concentration in water: (a) 0.25 wt\%; (b) 2 wt $\%$; (c) 4 wt $\%$; (d) 8 wt\%.

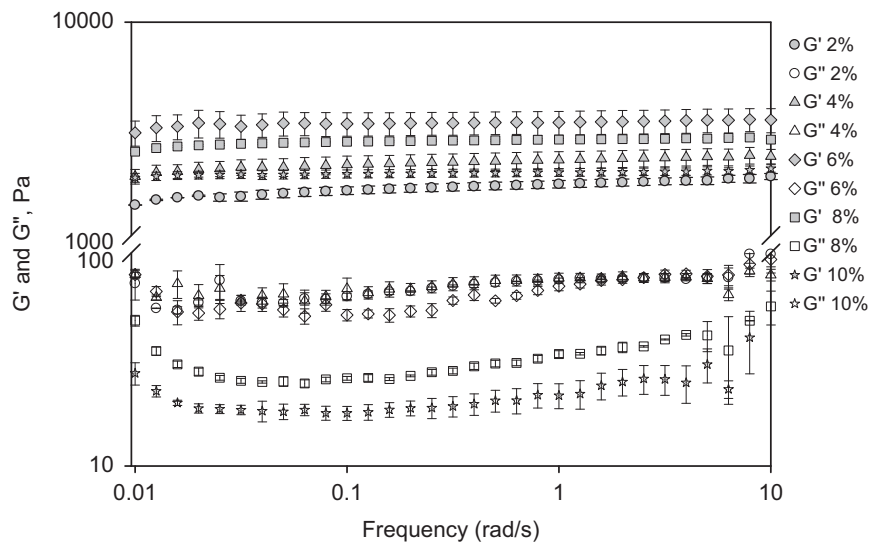

Fig. 2. Storage modulus, $G^{\prime}$, (a) and loss modulus, $G^{\prime \prime}$, (b), as function of the frequency of macrogels obtained at the variance of polymer concentration in water.

(8 and 10 wt\%) a decrease of both $G^{\prime}$ and $G^{\prime \prime}$ occurs. The $G^{\prime}$ increase for systems ranging from 2 to $6 \mathrm{wt} \%$ can be attributed to the increase of crosslinking density. Above $6 \mathrm{wt} \%$ the crosslinking density may further increase locally, but at the expense of the spatial network homogeneity yielding very compact but weakly interconnected clusters. Accordingly, $G^{\prime \prime}$ decreases: the reduction of dissipative phenomena, evidenced by the decrease of $G^{\prime \prime}$, can be explained with the relative easiness of mutual slipping of neighboring densely crosslinked clusters. The average molecular weight between two adjacent crosslinks, $M_{\mathrm{c}}$, and the average distance between crosslinks or mesh size, $\xi$, were calculated from well known equations derived from the general theory of rubber elasticity (Rubinstein and Colby, 2003; Flory, 1953; LoPresti et al., 2011) and reported in Table 1 . The lowest $M_{c}$ pertains to the macrogel at $6 \mathrm{wt} \%$. Increases in $M_{\mathrm{c}}$, as well as of network mesh size, for concentrations below this value may reflect a looser networked structure. Conversely, hydrogels at 8 and $10 \mathrm{wt} \%$ combine lower $\xi$ values with higher average molecular weight
Table 2

Mean hydrodynamic radius and standard deviation of nanogels obtained at different polymer concentrations.

\begin{tabular}{lllll}
\hline \multicolumn{5}{l}{ Av. $\boldsymbol{R}_{\mathbf{h}}, \mathbf{n m}$ (Std. Dev.) } \\
\cline { 2 - 5 } & $\mathbf{0 . 5}$ (Sol fract.) & $\mathbf{0 . 2 5}$ & $\mathbf{0 . 1}$ & $\mathbf{0 . 0 5}$ \\
\hline $\begin{array}{llll}\text { Not irradiated } \\
\text { Irradiated }\end{array}$ & $22(3)$ & $22(8)$ & $20(10)$ & $20(9)$ \\
& $36(8)$ & $60(14)$ & $15(4)$ & $13(4)$ \\
\hline
\end{tabular}

between adjacent crosslinks. In the aforementioned hypothesis of spatial heterogeneity of the networks for these two systems, the two average molecular parameters calculated from a bulk macromechanical behavior found the limit of their usefulness.

The RR plateau values are in well agreement with the above discussed hypothesis on the network organization of macrogels. The poorly developed network of the $2 \mathrm{wt} \%$ system, upon freeze-drying, collapses into a non-porous solid (see Fig. 2b) that cannot be fully rehydrated. The yet limited number of crosslinking points, in fact, reduces the polymer chains mobility, thus preventing full hydration of PVP polar groups and activation of the typical hydrogel swelling driving forces, such as capillary and osmotic forces (Peppas et al., 2006). The 4 and $6 \mathrm{wt} \%$ systems can be considered proper "wall to wall" macrogels that optimally combine chain elasticity and network permeability, thus ensuring "reversibility" of the freeze-drying/rehydration processes. Finally, 8 and $10 \mathrm{wt} \%$ systems show high RR values at plateau. For the postulated inhomogeneity in the distribution of crosslink points, the RR measured see the modest contribution of the highly cross-linked regions and a most conspicuous contribution of the looser tie zones.

The soluble part of the $0.5 \mathrm{wt} \%$ system and all the other systems produced at lower polymer concentration have been investigated through dynamic light scattering. In Table 2 the calculated average hydrodynamic radii and relative standard deviations for the scattering angle $\theta=90^{\circ}$ are reported. Values refer to systems that were subjected to filtration with $0.22 \mathrm{~mm}$ pore size filters and to four times dilution with distilled water. It 
Table 3

${ }^{13} \mathrm{C}\left\{{ }^{1} \mathrm{H}\right\} C P$-MAS NMR - proton spin lattice relaxation time in the rotating frame of hydrogels.



is worth noting that decay curves, here not shown for the sake of brevity, were the same for systems at different dilution ratios and before and after successive filtration with filters of 5, 1.2, 0.8 and $0.45 \mu \mathrm{m}$, thus indicating that all the systems are fairly stable colloids. Further filtration with $0.22 \mu \mathrm{m}$ leaves unaffected the decay curves of the 0.5 and $0.25 \mathrm{wt} \%$ formulations but causes a slight reduction in scattering light intensity for 0.1 and $0.05 \mathrm{wt} \%$ systems. Finally, measurements carried out at the variance of the scattering angle, in the range of $30-150^{\circ}$, lead always to the same average hydrodynamic diameters. Standard deviations are only slightly higher at the lowest value in the range. The 0.5 and 0.25 wt\% systems present nanoparticles with average hydrodynamic radii of 32 and $50 \mathrm{~nm}$, respectively, that are higher than those of the unirradiated PVP coils. This can be due to the occurrence of both intermolecular and intramolecular crosslinking. On the contrary, nanogels obtained from 0.1 and $0.05 \mathrm{wt} \%$ solutions have hydrodynamic radii lower than that of the corresponding unirradiated systems. In this case, intramolecular crosslinking may be the prevailing phenomenon, according to the already reported effect of polymer concentration on the nanogels size (Ulanski and Rosiak, 1999). No reduction of average weight molecular weight has been observed in these irradiation conditions, as determined by static light scattering.

In Table 3 the proton spin lattice relaxation times in the rotating frame $\left(\mathrm{T}_{1 \rho} \mathrm{H}\right)$ are reported, both for linear PVP and the lyophilized irradiated systems. It is known that $\mathrm{T}_{1 \rho} \mathrm{H}$ values are sensitive to the molecular motions, which occur in the $\mathrm{kHz}$ regions and are associated with cooperative polymer backbone rearrangements, which envelop the collective motions of a large number of monomer units (Boyer, 1968).

As a general consideration, the $\mathrm{T}_{1 \mathrm{\rho}} \mathrm{H}$ values are higher for linear PVP than the corresponding values for the irradiated ones. This indicates that the local motions of the linear polymer are more hindered, probably due to the higher packaging density attained in the solid state from the flexible linear PVP chains and establishment of extensive inter and intra-molecular interactions, such as water molecules mediated hydrogen bonding as well as electrostatic interactions between the negatively charged carbonyl groups and positively charged amino groups of different pyrrolidone rings formed by keto-enol tautomerization. Upon crosslinking, the reduced mobility of macromolecular segments makes the establishment of inter/intramolecular interactions in the solid state less efficient and $\mathrm{T}_{1 \rho} \mathrm{H}$ values are comparatively lower. A comparison among the different irradiated macrogels shows that increasing the polymer concentration, the $T_{1 \rho} \mathrm{H}$ values steadily increase, thus suggesting a monotonic increase of crosslinking density. These results support the interpretation of the rheological data, which showed for the $8 \mathrm{wt} \%$ system, a decrease of both $G^{\prime}$ and $G^{\prime \prime}$ values, with respect to the systems with lower polymer concentration. Finally, the data for the lyophilized nanogel $(0.25 \mathrm{wt} \%$ system $)$ indicates the formation of a faster relaxing structure with respect to the linear PVP with $\mathrm{T}_{1 \mathrm{\rho}} \mathrm{H}$ values close to the corresponding values for the 4 and $6 \mathrm{wt} \%$ macrogels. The very low relaxation times for $\mathrm{C}_{6}$ and $\mathrm{C}_{4}$ reflect a partial modification of chemical structure of PVP in these two positions, observed for the nanogels only, that will be the subject of a further dedicated paper.

\section{Conclusions}

It is well known that high dose-rate pulsed electron irradiation leads to either macrogels or nanogels depending on the polymer concentration in water. Here we have irradiated aqueous solutions of a commercial PVP at concentrations ranging from above (10 wt\%) to below (0.05 wt\%) its critical chain overlap concentration $\left(C^{*} \cong 1 \mathrm{wt} \%\right)$ with an integrated dose that is within the sterilization dose range (40 kGy). When gelation is macroscopic, thereby occurring mainly in virtue of inter-molecular crosslinking, the average crosslink density can be estimated by dynamicmechanical data. When the network crosslinking density is not homogeneous or when the network is nanoscalar in size this conventional approach is not applicable. Solid-state ${ }^{13} \mathrm{C}$ NMR has been applied to calculate the spin-lattice relaxation times in the rotating frame $\left(\mathrm{T}_{1 \rho} \mathrm{H}\right)$ for the different carbon atoms of the PVP repetitive unit, to observe that crosslinking is associated to a general reduction of relaxation times of the dry gels with respect to the solid linear polymer, due to the reduced stacking ability of macromolecules upon crosslinking. The spin-lattice relaxation times, that are associated to the mobility of molecular segments in the scale of nanometers, gradually increase with the increase of polymer concentration in water for macrogels, thus confirming more and more densely crosslinked networks. Conversely, at the higher concentrations in the range the average elastic moduli from rheological data decrease, as $G^{\prime}$ is mainly affected by the loss of spatial homogeneity at the scale of tens of nanometers, leading to highly unrealistic estimated average mesh size values. The same technique can be applied to the formed nanoscalar networks, or nanogels, generated at concentrations lower than $\mathrm{C}^{*}$, although particular care has to be paid to any eventual structural modification of the polymer. This aspect of high energy irradiation induced modification of the polymer will be specifically addressed in a dedicated paper owing to its fundamental and application-related importance.

\section{Acknowledgments}

Solid-state ${ }^{13} \mathrm{C}$ NMR experimental data were provided by Centro Grandi Apparecchiature - UniNetLab - Università di Palermo funded by P.O.R. Sicilia 2000-2006, Misura 3.15 Quota Regionale. 
This work was partially supported by the IAEA CRP: "Nanoscale radiation engineering of advanced materials for potential biomedical applications" and FW7 COST Action MP0701.

\section{References}

An, J.-C., 2009. Synthesis of poly(vinyl pyrrolidone) nanohydrogels by the template-assisted ionizing radiation. J. Ind. Eng. Chem. 15, 148-152.

Anseth, K.S., Bowman, C.N., Brennon-Peppas, L., 1996. Mechanical properties of hydrogels and their experimental determination. Biomaterials 17, 1647-1657.

Boyer, R.F., 1968. Dependence of mechanical properties on molecular motion in polymers. Polym. Eng. Sci., 161-185 (Society of Plastics Engineers, Inc.).

Chmielewski, A.G., Haji-Saeid, M., Ahmed, S., 2005. Progress in radiation processing of polymers. Nucl. Instrum. Methods Phys. Res. Sect. B 236, 44-54.

D’Errico, G., De Lellis, M., Mangiapia, G., Tedeschi, A., Ortona, O., Fusco, S. Borzacchiello, A., Ambrosio, L., 2008. Structural and mechanical properties of UV-photocrosslinked poly(vinyl-2-pyrolidone) hydrogels. Biomacromolecules 9, 231-240.

Dispenza, C., Ricca, M., LoPresti, C., Battaglia, G., La Valle, M., Giacomazza, D., Bulone, D., 2011. E-beam irradation and UV photocrosslinking of microemulsion-laden poly(N-vinyl-2-pyrrolidone) hydrogels for "in-situ" encapsulation of volatile hydrophobic compounds. Polym. Chem. 2, 192-202.

Flory, P.J., 1953. Principles of Polymer Chemistry. Cornell University Press, New York

Henke, A., Kadlubowski, S., Rosiak, J.M., Arndt, K.F., 2005. Radiation-induced crosslinking of polyvinylpyrrolidone-poly(acrylic acid) complexes. Nucl. Instrum. Methods Phys. Res. Sect. B 263, 391-398.
Kofonas, P., Cohen, R., 1997. Development of methods for quantitative characterization of network morphology in pharmaceutical hydrogels. Biomaterials 18 , 1361-1369.

LoPresti, C., Vetri, V., Ricca, M., Foderà, V., Tripodo, G., Spadaro, G., Dispenza, C., 2011. Pulsatile protein release and protection using radiation-crosslinked polypeptide hydrogel delivery devices. React. Funct. Polym. 71, 155-167.

Lugao, A.B., Malmonge, S.M., 2001. Use of radiation in the production of hydrogels. Nucl. Instrum. Methods Phys. Res. Sect. B 185, 37-42.

Peppas, N.A., Hilt, J.Z., Khademhosseini, A., Langer, R., 2006. Hydrogels in biology and medicine: from molecular principles to bionanotechnology. Adv. Mater. $18,1345-1360$.

Ricca, M., Foderà, V., Giacomazza, D., Leone, M., Spadaro, G., Dispenza, C., 2010. Probing the internal environment of PVP networks generated by irradiation with different sources. Colloid Polym. Sci. 288, 969-980.

Rosiak, J.M., Olejniczak, J., 1993. Medical applications of radiation formed hydrogels. Radiat. Phys. Chem. 42, 903-906.

Rosiak, J.M., Ulanski, P., Pajewski, L.A., Yoshii, F., Makuuchi, K., 1995. Radiation formation of hydrogels for biomedical purposes. Some remarks and comments. Radiat. Phys. Chem. 46, 161-168.

Rosiak, J.M., Yoshii, F., 1999. Hydrogels and their medical applications. Nucl. Instrum. Methods Phys. Res. Sect. B 151, 56-64.

Rubinstein, M., Colby, R., 2003. Polymer Physics. Oxford University Press, New York.

Stepanek, P., 1993. Data analysis in dynamic light scattering. In: Brown, W. (Ed.), Dynamic Light Scattering. Oxford University, Oxford, UK, pp. 177-240.

Ulanski, P., Rosiak, J.M., 1999. The use of radiation technique in the synthesis of polymeric nanogels. Nucl. Instrum. Methods Phys. Res. Sect. B 151, 356-360. 